Mon. Not. R. Astron. Soc. 000, 000-000 (0000) Printed 15 August $2007 \quad$ (MN LATEX style file v2.2)

\title{
Spectroscopy of planetary mass brown dwarfs in Orion
}

\author{
P.W.Lucas ${ }^{1}$, D.J.Weights ${ }^{1}$, P.F.Roche ${ }^{2}$, F.C.Riddick ${ }^{3}$ \\ ${ }^{1}$ Dept. of Physical Sciences, University of Hertfordshire, College Lane, Hatfield AL10 9AB, England. \\ email: pwl@star.herts.ac.uk \\ ${ }^{2}$ Astrophysics, Physics Dept., University of Oxford, 1 Keble Road, Oxford OX1 3RH, England. \\ ${ }^{3}$ Dept. of Astronomy \&s Astrophysics, Penn State University, 525 Davey Lab, University Park, PA 16802, USA.
}

15 August 2007

\begin{abstract}
We report the results of near infrared spectroscopy of 11 luminosity selected candidate planetary mass objects (PMOs) in the Trapezium Cluster with Gemini South/GNIRS and Gemini North/NIRI. 6 have spectral types $\geq M 9$, in agreement with expectations for PMOs. 2 have slightly earlier types, and 3 are much earlier types which are probably field stars. $4 / 6$ sources with types $\geq$ M9 have pseudo-continuum profiles which confirm them as low gravity cluster members. The gravity status of the other cool dwarfs is less clear but these remain candidate PMOs. The derived number fraction of PMOs with $\mathrm{M}=3-15 \mathrm{M}_{\text {Jup }}$ is $1-14 \%$, these broad limits reflecting the uncertainty in source ages. However, the number fraction with $\mathrm{M}<20 \mathrm{M}_{\text {Jup }}$ is at least $5 \%$. These detections add significantly to the body of evidence that a planetary mass population is produced by the star formation process.
\end{abstract}

Key words: stars: low mass, brown dwarfs; stars: formation

\section{INTRODUCTION}

In recent years there have been several reported detections of free floating brown dwarfs with planetary masses in very young galactic clusters (eg. Oasa, Tamura \& Sugitani 1999; Lucas \& Roche 2000; Zapatero Osorio et al.2000; Allers et al.2006; Jayawardhana \& Ivanov 2006). Many of these candidates are not yet confirmed as cluster members with the low temperatures predicted by theoretical models. However, at least 1 confirmed PMO probably has $\mathrm{M}<10 \mathrm{M}_{J u p}$ : Cha 1109-7734 (Luhman et al.2005). Here we adopt a planetary mass threshold of $15 \mathrm{M}_{\text {Jup }}\left(0.014 \mathrm{M}_{\odot}\right)$ in order to include sources on the deuterium burning limit, which is at 13$14 \mathrm{M}_{J u p}$ for solar abundance (Chabrier et al.2000; Burrows et al.1997) but may be marginally higher in Orion, which has a low D/H ratio (see O'Dell 2004 and references therein).

Lucas, Roche \& Tamura (2005) (hereafter LRT) detected 33 candidate PMOs in a deep imaging survey of the Trapezium Cluster, and showed that $<13 \%$ of cluster members have masses in the range $3-13 \mathrm{M}_{\text {Jup }}$. These masses were estimated from source luminosities and an assumed age of $1 \mathrm{Myr}$, using the isochrone of the Lyon group. Here we present spectra of a sample of candidates chosen from LRT and Lucas \& Roche (2000) in an attempt to constrain the inital mass function (IMF) at planetary masses. In Section 2 the observations are described. In Section 3 we determine spectral types and surface gravity status and assess cluster membership. We then compare the results with the predictions of pre-main sequence models and discuss the IMF.

\section{OBSERVATIONS}

Near infrared spectra of PMO candidates in Orion were obtained in two observing programmes: one with GNIRS at Gemini South Observatory and one in classical mode with NIRI at Gemini North Observatory. The GNIRS data were taken in queue mode between November 2004 and November 2005. The NIRI data were obtained in the classical observing mode on 5-8 December 2003.

Useful results were obtained for 11 candidate PMOs (see Table 1). All are located in the outer parts of the Trapezium Cluster where the pervasive nebulosity is relatively faint. They were selected for low extinction $\left(A_{V} \lesssim 5\right.$ mag) which reduces the chance of observing background stars (see LRT). The GNIRS sample consisted of 7 candidate PMOs from LRT. With NIRI we observed 5 candidate PMOs, 4 selected from LRT and 1 (022-115) from Lucas \& Roche (2000). For one of these 5 candidates, 188-658, the data were of poor quality, so it was reobserved with GNIRS. The GNIRS data were taken with a short slit, so only one source could be observed at a time. Slit orientations which minimise the local nebulosity gradient in were chosen. The long slit of the NIRI spectrograph was oriented so as to include 2 or 3 sources simultaneously. Hence, an additional selection criterion, for the NIRI sources only, was a very weak local nebulosity gradient, in order to minimise the effects of the structured background on the extracted spectra. In both programmes the background emission was subtracted using a $3^{\prime \prime}$ nod between 2 positions along the slit at intervals of $2-4$ minutes. The 


\begin{tabular}{ccccccc}
\multicolumn{6}{c}{ Table 1 - Source List and Integration Times } \\
Source* & $\mathrm{K}$ & $\mathrm{H}$ & $\mathrm{J}$ & $\mathrm{A}_{V}$ & $\begin{array}{c}\text { Data } \\
\text { Type }\end{array}$ & $\begin{array}{c}\text { Time } \\
(\mathrm{min})\end{array}$ \\
& & & & & & $\mathrm{H}, \mathrm{K}$ \\
\hline $152-717$ & 17.61 & 18.39 & 19.38 & 3.7 & GNIRS & 120,120 \\
$188-658$ & 17.87 & 18.34 & 19.12 & 0.8 & GNIRS & 132,132 \\
$137-532$ & 17.20 & 18.27 & 19.39 & 5.3 & GNIRS & 120,120 \\
$092-532$ & 17.71 & 18.22 & 19.09 & 2.4 & GNIRS & 56,56 \\
$107-453$ & 17.94 & 18.71 & 19.46 & 0.3 & GNIRS & 252,252 \\
$016-430$ & 17.89 & 18.94 & 19.98 & 2.4 & GNIRS & 72,72 \\
$057-247$ & 17.90 & 18.86 & 19.92 & 2.8 & GNIRS & 96,96 \\
$183-729$ & 17.24 & 17.38 & 18.05 & 1.3 & NIRI & 32,124 \\
$199-617$ & 17.64 & 18.96 & 20.17 & 5.2 & NIRI & 56,180 \\
$205-610$ & 17.16 & 17.81 & 18.52 & 1.1 & NIRI & 56,180 \\
$022-115$ & - & 18.41 & 19.28 & 2.1 & NIRI &,- 184
\end{tabular}

* Source names are coordinate based, following O'Dell \& Wong (1996). 022-115 was listed as 023-115 in Lucas et al.(2001) due to a small astrometric error.

GNIRS data were taken in cross dispersed mode with the 32 lines/mm grating. Here we focus on the data in 2 orders covering the $\mathrm{H}(1.65 \mu \mathrm{m})$ and $\mathrm{K}(2.2 \mu \mathrm{m})$ bands, where the data quality is best. Slit widths of $0 .{ }^{\prime \prime} 45$ or 0. " 675 were used, depending on the seeing conditions. The spectral resolution was $\mathrm{R}=\lambda / \Delta \lambda \approx 950$ in the $\mathrm{H}$ and $\mathrm{K}$ bands with the $0 .{ }^{\prime \prime} 675$ slit and $R \approx 1400$ with the $0 . " 45$ slit. The NIRI spectra were taken with a $0 .{ }^{\prime \prime} 75$ slit and the $\mathrm{f} / 6$ camera. The $\mathrm{K}$ band grism was used for most observations but the $\mathrm{H}$ band grism was used to take some data at relatively high airmass. The resolution was $\mathrm{R} \approx 520$ for all the NIRI data.

The data were reduced with the IRAF software package. The $\mathrm{OH}$ sky lines were used to wavelength calibrate the GNIRS spectra, and argon lamp spectra were used for the NIRI data. F and G-type stellar standards were used to calibrate the throughput. The extracted spectra were cleaned of narrow noise spikes attributable to bad pixels, cosmic rays and time variable telluric $\mathrm{OH}$ emission lines, using independent subsets of the data to ensure that genuine features were not discarded. Residual background features caused by 3 to 5 of the brightest $\mathrm{HI}, \mathrm{H}_{2}$ or HeI lines (see Marconi et al. 1998) were also fitted and subtracted.The independent subsets were then coadded and dereddened with the DEREDDEN task in IRAF to produce the final results.

\section{RESULTS AND ANALYSIS}

\section{$3.1 \quad$ Spectral Typing}

In Fig. 1 we present the GNIRS and NIRI $\mathrm{H}$ and $\mathrm{K}$ band spectra for the 8 sources with strong water vapour absorption. Fig. 1 also includes a K band spectrum of 022-115, 1 of the 3 sources with little or no steam absorption, and 2 high quality spectra from the template samples described below. Steam absorption causes broad depresssions on either side of the peaks near $1.675 \mu \mathrm{m}$ in the $\mathrm{H}$ band and $2.20 \mu \mathrm{m}$ in the $\mathrm{K}$ band. In contrast $022-115$ shows a smooth Rayleigh-Jeans continuum, which is typical of normal field stars with spectral type $\leq \mathrm{M} 3$, eg. Leggett et al.(1996). Cubic spline fits to the plotted spectra generated by the IRAF task SPLOT are overplotted for the sources in Orion. These fits (5th order for $\mathrm{H}$ band and 6th order for $\mathrm{K}$ band) represent the pseudo-continuum quite well, without following the narrower structures in the data, which are simply due to noise.

In Table 2 we provide the spectral types derived from four separate steam absorption indices, which were applied to the pseudo-continuum fits. The indices were calibrated using a template sample of 38 young $M$ dwarfs for which high quality 1-2.5 $\mu \mathrm{m}$ spectra taken with the SpeX instrument on the NASA Infrared Telescope Facility were provided by K.Luhman (see Luhman 2006; Muench et al., in prep). A second template sample, composed of late M and L type field dwarfs with high quality infrared spectra from the same instrument, was also provided by K.Luhman. The spectral types of the young $M$ dwarfs were defined at optical wavelengths using the dwarf/giant average system of Luhman (1999). All are located in the Chamaeleon or Taurus star formation regions, which have similar ages to the Trapezium Cluster. The indices are defined by the following flux ratios: $\mathrm{WH}=\left(\mathrm{F}_{\lambda}(1.525)\right) / \mathrm{F}_{\lambda}(1.675)$. $\mathrm{QH}=\mathrm{F}_{\lambda}(1.57) / \mathrm{F}_{\lambda}(1.675)\left(\mathrm{F}_{\lambda}(1.77) / \mathrm{F}_{\lambda}(1.675)\right)^{1.282}$.

$\mathrm{WK}=\mathrm{F}_{\lambda}(2.05) / \mathrm{F}_{\lambda}(2.23)$.

$\mathrm{QK}=\left(\mathrm{F}_{\lambda}(2.05) / \mathrm{F}_{\lambda}(2.2)\right)\left(\mathrm{F}_{\lambda}(2.34) / \mathrm{F}_{\lambda}(2.2)\right)^{1.262}$. The median $\mathrm{F}_{\lambda}$ values within $0.02 \mu \mathrm{m}$ intervals were used, eg. 1.515-1.535 $\mu \mathrm{m}$ for $\mathrm{F}_{\lambda}$ (1.525). Relations between spectral type and index were fitted as cubic polynomials for all 4 indices. These have a typical scatter of 0.5 subtypes. The properties of the indices are fully described by Weights (2006) and Weights et al.(in prep). To summarize, the QH and QK indices are reddening independent measures of the water absorption on both sides of the $\mathrm{H}$ and $\mathrm{K}$ band flux peaks respectively. Similar indices were used by Wilking, Greene \& Meyer (1999) and Lucas et al.(2001). The WH and WK indices measure the steam absorption only on the short side of the $\mathrm{H}$ and $\mathrm{K}$ bands respectively and require accurate $A_{V}$ values in Table 1 . However, they provide an independent check on the results and WK is less affected than QK by any veiling due to hot circumstellar dust on the long side of the $\mathrm{K}$ bandpass.

It should be noted that steam absorption strengthens rather slowly between M7 and M9 in the Luhman sample. The QK index discriminates best between these types. The quoted final types in Table 2 are based on comparison of the four indices and consideration of any source-specific aspects of the data (see notes to the Table). The final types are also influenced by the GNIRS J band spectra for the first 7 objects in the table. The J band spectra (not shown) all exhibit a strong water absorption edge at $1.34 \mu \mathrm{m}$. Visual comparison with a large sample of field $M$ dwarfs provided by S.Leggett (private comm.) shows that this is sufficient to classify these 7 objects as >M5 (see eg. Leggett et al.1996). We do not attempt to gain more precise information from the $\mathrm{J}$ band data, owing to the low signal to noise and the complicating effect of telluric water absorption near $1.34 \mu \mathrm{m}$.

\subsection{Surface Gravity and Cluster Membership}

PMOs with the canonical 1 Myr age of Trapezium cluster members are predicted to have surface gravities 50 to 100 times lower than field dwarfs of similar spectral type, since they have not yet contracted to the stable radius $\left(\mathrm{R} \approx 0.1 \mathrm{R}_{\odot}\right)$ that is supported by degeneracy pressure in mature field dwarfs. The clearest indicator of cluster membership in very young sources is the detection of low sur- 

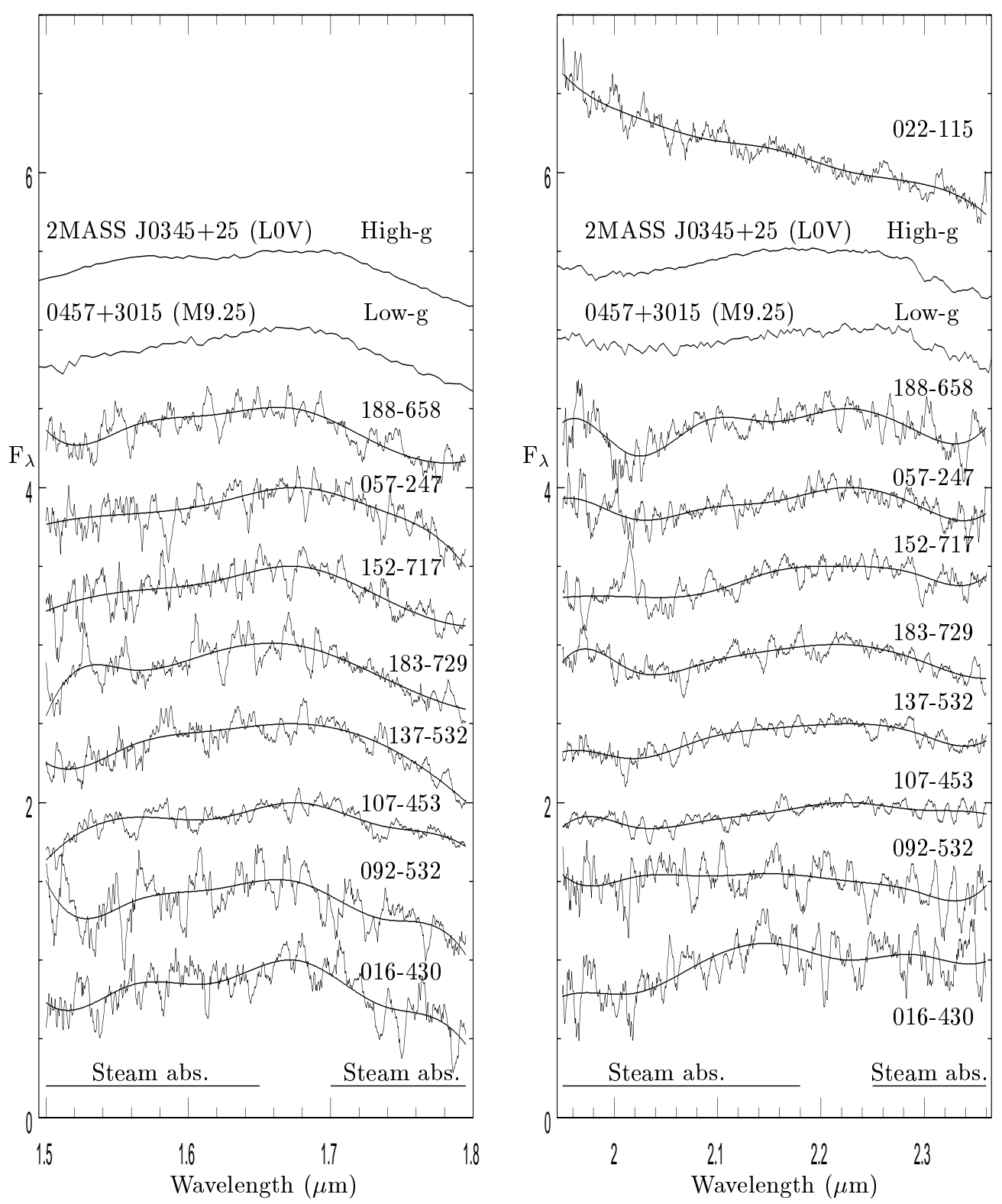

Figure 1. Spectra of the 8 Trapezium sources with strong steam absorption bands (the bottom 8 spectra) and 1 Trapezium source (022-115) with little or no steam absorption (top of right hand panel). An 11 pixel boxcar smoothing was applied. The fits to the pseudo continuum are overplotted as solid curves. Template spectra from the low-g and high-g samples provided by K.Luhman (see text) are also shown to illustrate the differences expected between low-g cluster members and cool high-g field dwarfs. Spectra are normalised to unity at the value of the curves at $1.675 \mu \mathrm{m}$ and $2.23 \mu \mathrm{m}$ and are vertically displaced by multiples of 0.5 units for clarity.

face gravity (low-g) features in the optical or infrared spectrum (eg. McGovern et al. 2004). The most easily detected low-g feature is the triangular profile of the $\mathrm{H}$ band pseudocontinuum, which peaks at $\sim 1.675 \mu \mathrm{m}$ in an $\mathrm{F}_{\lambda}$ plot, identified by Lucas et al.(2001); see also Natta et al.(2002), Meeus \& McCaughrean (2005). This contrasts with the flat topped profile observed in late $\mathrm{M}$ and $\mathrm{L}$ type high gravity (highg) field dwarfs, a difference attributed by Kirkpatrick et al.(2006) to less collision induced $\mathrm{H}_{2}$ absorption in low-g objects. In addition, the following systematic differences between the pseudo continua of low-g and high-g objects are observed in the $\mathrm{K}$ band in the template samples. (1) $\mathrm{F}_{\lambda}$ spectra of very young late $M$ type brown dwarfs have a typically quite flat maximum between 2.18 and $2.28 \mu \mathrm{m}$ (eg. Luhman,
Peterson \& Megeath 2004) whereas field dwarfs with types M7-L7 all have a maximum between 2.14-2.18 $\mu \mathrm{m}$ and decline slowly from $\lambda=2.18-2.29 \mu \mathrm{m}$. This decline is more pronounced at mid-late L types. (2) The CO ( $\mathrm{v}=2-0)$ absorption trough located at $\lambda \geq 2.29 \mu \mathrm{m}$ is weaker in very young brown dwarfs with types M7-M9.5 than in the field dwarfs with equivalent (optically determined) spectral type.

The surface gravity status of the 8 cool Orion dwarfs is indicated in Table 2. For some sources, eg. 152-717 and 057-247, the triangular $\mathrm{H}$ band profile is obvious from visual inspection. However, for several candidates the low signal to noise required a statistical test to determine whether the $\mathrm{H}$ and $\mathrm{K}$ band spectra data are more consistent with low- $\mathrm{g}$ template spectra than high-g field dwarf templates. For each 


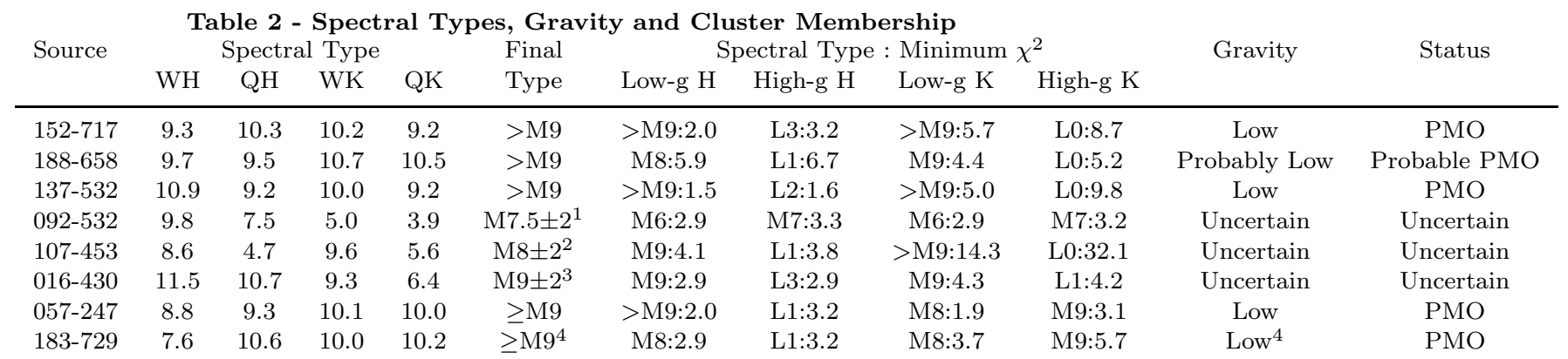

Source notes: (1) greater weight was given to the H band indices for 092-532, owing to the poorer quality of the K band spectrum. (2) The QK value was neglected, since 107-453 appears to have a K band excess, judging by the JHK fluxes and the K band continuum profile. (3) 016-430 shows a significant discrepancy between the WK and QK results, probably due to the low signal to noise of the data. (4) The K band results were given more weight than $\mathrm{H}$ band for 183-729, due to the longer integration time. 183-729 was found to have low gravity and type M8.75 \pm 1.5 in the optical spectrum of Riddick (2006).

candidate reduced $\chi^{2}$ values were calculated separately for the smoothed $\mathrm{H}$ and $\mathrm{K}$ band spectra using young dwarf and field dwarf spectra from the template samples. Best fits were identified by $\chi^{2}$ minimisation for a range of spectral types: M4 to M9.5 for the young dwarf templates and M4 to L3 for the field dwarf templates. The uncertainties in $F_{\lambda}$ at each wavelength were estimated using the scatter calculated in narrow wavelength bins and were propagated through to the smoothed spectra. This will underestimate the errors if there are also systematic effects, e.g. correction for atmospheric absorption. Hence, the absolute values of $\chi^{2}$ are difficult to interpret and are not used to derive formal probabilities. However, relative values can be used to determine whether high-g or low-g templates provide the better fits.

The $\chi^{2}$ results show that 4 sources have spectra that are more consistent with low-g late $\mathrm{M}$ or early $\mathrm{L}$ type templates than high-g templates in the $\mathrm{H}$ and/or $\mathrm{K}$ bands: 057-247, 152-717, 137-532, and 183-729 (Table 2). Low-g status is also probable for 188-658, though the result is less clear. For 107-453, the K-band fits are poor owing to an unusually high flux at $\lambda>2.2 \mu \mathrm{m}$. This may be due to circumstellar dust emission, which would suggest that it is a young object. The $\chi^{2}$ values for the best fits to 016-430 and 092-532 are not significantly different for the low- and high-gravity templates. The 3 sources exluded from Table 2 are almost certainly field stars, with spectral types <M5.

It is instructive to calculate the expected number of high-g field dwarfs within the $26 \operatorname{arcmin}^{2}$ area of the LRT survey with similar spectral types and fluxes to the PMO candidates. The typical absolute magnitudes for single M9 and L5 field dwarfs are $\mathrm{M}_{K}=10.29,11.61$ respectively (Kirkpatrick et al. 2000) on the MKO system. Hence M9 and L5 field dwarfs would appear fainter than all the sources in Table 1 at distances, $d>339$ pc (M9) or 185 pc (L5). Spectral types $>$ L5 were ruled out by the $\chi^{2}$ fits. M9-L5 type field dwarfs have effective temperatures $1700<T_{\text {eff }}<2400 \mathrm{~K}$ (Golimowski et al.2004) and a space density in the range 0.0067-0.0145 $\mathrm{pc}^{-3}$ (Burgasser 2004). Multiplying the space density for each sub-type by the appropriate volume and summing the results, an expected contamination of 0.36 $0.72 \mathrm{M} 9-\mathrm{L} 5 \mathrm{~V}$ single systems was calculated. This rises to 0.61-1.22 objects when unresolved binaries are included, assuming an incidence of 25\% (Basri \& Reiners 2006) and equal luminosity for both components. If we conservatively assume a Poisson distribution with a mean of 1.22 and ne-

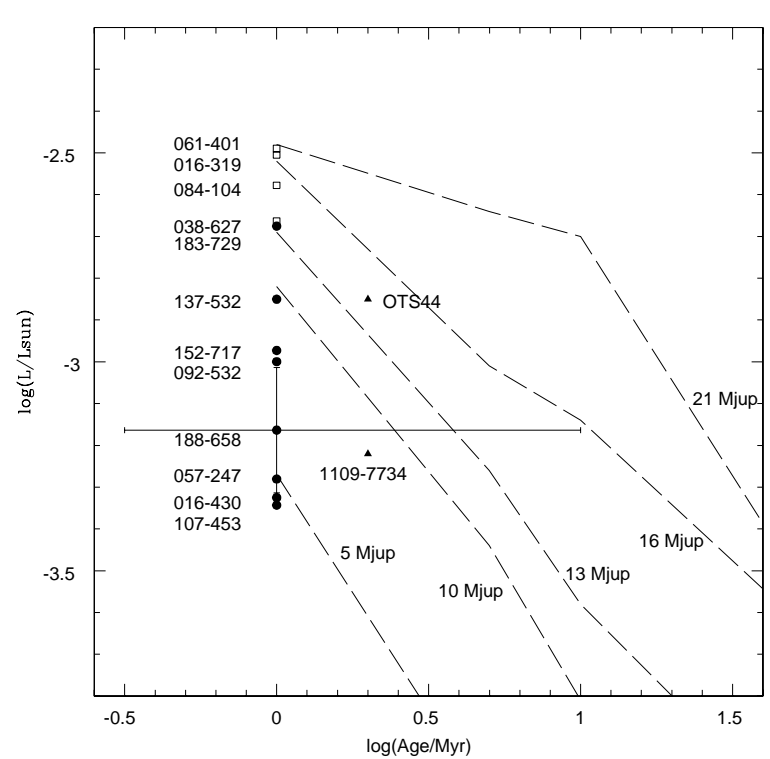

Figure 2. Luminosity vs. age. The sources in Table 2 are plotted, with assumed ages of $1 \mathrm{Myr}$ (filled circles). The theoretical evolutionary tracks of Chabrier et al.(2000) are plotted as dashed lines for various masses. For comparison we plot the 2 very low mass sources OTS44 and Cham 1109-7734 in the Chamaeleon star formation region (filled triangles) and the 4 least luminous Trapezium sources confirmed as cool dwarfs in Lucas et al.(2001) (open squares). Error bars are shown for only 1 source to aid clarity but are similar for all sources.

glect candidates which have not yet been observed, the probability that all 6 sources in Table 2 with types $\geq$ M9 are field dwarfs is $0.2 \%$ and the probability that more than 3 are field dwarfs is $3.6 \%$. When M6-M8 objects are included, the expected number of contaminating objects rises by a factor of $\sim 2$, so it is quite possible that 1 or 2 of the sources whose gravity status is uncertain are field dwarfs.

\subsection{Comparison with model isochrones}

We estimate the masses of the cluster members by comparison with the model isochrones of Chabrier et al.(2000). The masses can be derived from the isochrone using (i) luminosity and assumed age (see LRT), or (ii) temperature and assumed age. In theory, both mass and age can be derived from luminosity and temperature using the HR diagram but this 
method is too temperature sensitive at $2000-2500 \mathrm{~K}$ to produce useful results. If an age of $1 \mathrm{Myr}$ is assumed, method (i) gives masses $\mathrm{M} \leq 13 \mathrm{M}_{J u p}$ in every case. Spectral types $\geq \mathrm{M} 9$ correspond to $\mathrm{T}_{e} f f \leq 2400 \mathrm{~K}$, using the temperature scale of Luhman et al.(2003) that has been extensively applied in star formation regions. At $1 \mathrm{Myr}$, method (ii) then indicates $\mathrm{M} \leq 15 \mathrm{M}_{\text {Jup }}$. Hence the luminosities and temperatures agree with expectations for PMOs with an age of $\sim 1 \mathrm{Myr}$. If an age of $10 \mathrm{Myr}$ is assumed then the masses derived from method (i) increase somewhat, as shown in Fig.2. However, $\mathrm{M} \leq 20 \mathrm{M}_{J u p}$ in every case, and we find $\mathrm{M} \leq 15 M_{J u p}$ for the confirmed cluster member 057-257 and the likely cluster members 188-658 and 016-430. For method (ii) a $10 \mathrm{Myr}$ age also gives $\mathrm{M} \leq 20 \mathrm{M}_{J u p}$ for the 6 sources with spectral types $\geq$ M9. Since the cluster appears to contain fewer PMOs than more massive brown dwarfs (see LRT) any age spread in the cluster would be expected to bias our luminosity selected sample towards larger ages than the cluster average. Most researches have shown that the great majority of stellar and substellar cluster members have ages less than a few Myr(Riddick 2006; Lucas et al.2001; Hillenbrand 1997; Lada et al.2004) However, some studies suggest a broader age spread. Eg. Palla et al.(2005) detected lithium depletion in some low mass stars and proposed that star formation began in the cluster at a low rate $\sim 10 \mathrm{Myr}$ ago and accelerated toward the present day.

\subsection{The Initial Mass Function}

Since 10 Myr ages are possible for the PMO candidates, it is not yet proven that any have mass $\mathrm{M}<10 M_{\text {Jup }}$. However, even at age $10 \mathrm{Myr}$ at least 1 source is confirmed with $\mathrm{M}<15 M_{\text {Jup }}$, along with 2 other likely cluster members. The model isochrones also become highly uncertain at ages less than a few Myr (Baraffe et al.2002). However recent dynamical mass measurements of an eclipsing binary brown dwarf in the Trapezium cluster agree well with the predictions of the 1 Myr isochrone (Stassun, Mathieu \& Valenti 2006) and less direct mass estimates based on surface gravities (Mohanty et al.2004) are also fairly consistent with the predictions for very young brown dwarfs. Hence, at present the evidence suggests the model isochrones yield approximately correct masses at ages as low as 1 Myr.

This dataset therefore adds significantly to the evidence that free floating PMOs exist in very young clusters, extending the results of Lucas et al.(2001) to lower luminosities. In a sample of 11 PMO candidates we have 4 confirmed cluster members, 1 very probable member, 3 sources with uncertain status, and 3 field stars. Assuming an age of $1 \mathrm{Myr}$, LRT found that PMO candidates with $\mathrm{M}=3-13 \mathrm{M}_{\text {Jup }}$ number $13.2 \%$ of the population. Considering only the 7 sources whose status is clear, and assuming a $1 \mathrm{Myr}$ age and Poisson statistics, a number fraction of confirmed PMOs of $7.5 \% \pm 2.7 \%$ is simplistically derived. However, $\sim 50 \%$ of the candidates of LRT lay in the 3-5 $\mathrm{M}_{\text {Jup }}$ mass bin, which is under-represented in this sample (see Fig.2) but may contain a higher fraction of field stars. In addition, since the bias towards ages $>1 \mathrm{Myr}$ at very low luminosities is not quantified, the number fraction of PMOs can only be loosely constrained. However, we can say that the PMO number fraction in the range $3-15 \mathrm{M}_{J u p}$ is almost certainly between $1 \%$ and $14 \%$, and the fraction with $\mathrm{M}<20 \mathrm{M}_{J u p}$ is $>5 \%$.

\section{ACKNOWLEDGEMENTS}

We thank Kevin Luhman for supplying the template spectra used to calibrate the Orion spectra. This paper is based on observations obtained in programmes GS-2004B-Q-11 and GN-2003B-C-1 at the Gemini Observatory, which is operated by the Association of Universities for Research is Astronomy, Inc., under a cooperative agreement with the NSF on behalf of the Gemini partnership: the National Science Foundation (USA), the Particle Physics and Astronomy Research Council (UK), the National Research Council (Canada), CONICYT (Chile), the Australian Research Council (Australia), CNPq (Brazil) and CONICET (Argentina).

\section{REFERENCES}

Baraffe I., Chabrier G., Allard F., Hauschildt P.H., 2002, A\&A, 382,563

Basri G., Reiners A., 2006, AJ 132, 663

Burgasser A. 2004, ApJS, 155, 191

Chabrier G., Baraffe I., Allard F., Hauschildt P. 2000, ApJ 542, 464

Golimowski D.A., Leggett S.K., Marley M.S., Fan X., Geballe T.R., Knapp G.R., Vrba F.J., Henden A.A., Luginbuhl C.B., Guetter H. H. and 9 coauthors, AJ 2004, 127, 3516

Hillenbrand L.A. 1997, AJ 113,1733

Jayawardhana R., \& Ivanov V.D., 2006, ApJ 647, L167

Kirkpatrick J.D., Reid I.N., Liebert J., Gizis J.E., Burgasser A.J., Monet D.G., Dahn C.C., Nelson B., Williams R,J., 2000, AJ 120, 447

Kirkpatrick J.D., Barman T.S., Burgasser A.J., McGovern M.R., McClean I.S., Tinney C.J., Lowrance P.J. 2006, ApJ, 639, 1120

Lada C.J., Muench A.A., Lada E.A., Alves J.F. 2004, AJ, 128, 1254

Leggett S.K., Allard F., Berriman G., Dahn C.C., 1996, ApJS 104, 117

Lucas P.W., Roche P.F., 2000, MNRAS 314, 858

Lucas P.W., Roche P.F., Allard F., Hauschildt P.H. 2001, MNRAS 326, 695

Lucas P.W., Roche P.F., Tamura M., 2005, MNRAS 361, 211

Luhman K.L., 1999, ApJ, 525, 466

Luhman K.L., Stauffer J.R., Muench A.A., Rieke G.H., Lada E.A., Bouvier J., Lada C.J., 2003, ApJ 593, 1093

Luhman K.L., Peterson D.E., Megeath S.T. 2004, ApJ 617, 565

Luhman K.L., Adame L., D'Alessio P., Calvet N., Hartmann L., Megeath S.T., Fazio G.G., 2005, ApJ 635, L93

Luhman K.L., 2006, ApJ 645, 676

McGovern M.R., Kirkpatrick J.D., McLean I.S., Burgasser A.J., Prato L., Lowrance P.J., 2004, ApJ 600, 1020

Meeus G., McCaughrean M.J., 2005, AN 326, 977

Mohanty S., Jayawardhana R., Basri G. 2004, ApJ 609, 885

Natta A., Testi L., Comeron F., Oliva E., D'Antona F.D., Baffa

C., Comoretto G., Gennari S., 2002, A\&A 393, 597

Oasa Y., Tamura M., \& Sugitani K. 1999, ApJ 526,336

O'Dell C.R., 2001, ARA\&A 39, 99

O'Dell C.R., Wong K., 1996, AJ 111, 846

Palla F., Randich S., Flaccomio E., Palla R., 2005, ApJ 626, L49 Riddick F.C., 2006, PhD thesis, Oxford University.

Stassun K.G., Mathieu R.D., Valenti J.A., 2006, Nature 440, 311 Weights D.J., 2006, http://star-www.herts.ac.uk/ dweights Zapatero Osorio M.R., Béjar, V.J.S., Martin E.L., Rebolo R., Barrado y Navascués D., Bailer-Jones C.A.L., Mundt R., Science 290, 103 